\title{
Algorithm for constructing counterexamples to the Kalman problem
}

Leonov G.A., Bragin V.O., Kuznetsov N.V.

Draft 12

\begin{abstract}
The method of harmonic linearization and describing function method, numerical methods, and the applied bifurcation theory together discover new opportunities for analysis of hidden periodic oscillations (with basin of attraction which does not contain neighborhoods of equilibria) of control systems. In the present paper new algorithms for construction of counterexamples to Aizerman's conjecture and Kalman's conjecture is suggested.
\end{abstract}

Keywords: Hidden oscillation, describing function method justification, harmonic balance, absolute stability, nonlinear control system, Aizerman problem, Aizerman conjecture, Kalman problem, Kalman conjecture

\section{Introduction. Aizerman and Kalman conjectures}

Consider a system

$$
\frac{d x}{d t}=P x+q \varphi\left(r^{*} x\right), \quad x \in \mathbb{R}^{n}
$$

where $P$ is a constant $n \times n$-matrix, $q, r$ are constant $n$-dimensional vectors, $*$ is an operation of transposition, $\varphi(\sigma)$ is a continuous piecewise-differentiable scalar function, and $\varphi(0)=0$. Suppose that for all $k \in\left(\mu_{1}, \mu_{2}\right)$ a zero solution of system (1.1) with $\varphi(\sigma)=k \sigma$ is asymptotically stable in the large (i.e. a zero solution is Lyapunov stable and any solution of system (1.1) tends to zero as $t \rightarrow \infty$. In other words, a zero solution is a global attractor of system (1.1)).

In 1949 M.A. Aizerman (Aizerman (1949)) has formulated the following conjecture: all systems (1.1), satisfying the property

$$
k_{1} \sigma<\varphi(\sigma)<k_{2} \sigma \quad \sigma \neq 0
$$

are stable in the large.

The necessary criteria of absolute stability Leonov et al. (1996) contradict this hypothesis.

In 1957 R.E. Kalman has formulated a similar hypothesis (Kalman (1957)) with more restrictive condition (Kalman's problem or Kalman's conjecture): if at the points of differentiability $\varphi(\sigma)$ the condition

$$
\mu_{1}<\varphi^{\prime}(\sigma)<\mu_{2}
$$

is satisfied, then system (1.1) is stable in the large.

It is well known that this hypothesis is valid for $n=2$ and $n=3$ Leonov et al. (1996).

The only widely cited in literature counterexample to this hypothesis is due to Fitts Fitts (1966). It is obtained by numerical modeling of system (1.1) for $n=4$ with the transfer function

$$
W(p)=\frac{p^{2}}{\left[(p+\beta)^{2}+0.9^{2}\right]\left[(p+\beta)^{2}+1.1^{2}\right]}
$$

\footnotetext{
${ }^{1}$ Nikolay V. Kuznetsov, nkuznetsov239 at gmail.com (correspondence author)

${ }^{2} \mathrm{PDF}$ slides http://www.math.spbu.ru/user/nk/PDF/Hidden-oscillation-Absolute-stability-Aizerman-problem-Kalman.pdf
} 
and the cubic nonlinearity $\varphi(\sigma)=k \sigma^{3}$. In these numerical experiments for certain values of parameters $\beta$ and $k$ the periodic solutions of system (1.1) were obtained. However it was shown Barabanov $(1982,1988)$ that for some parameters the results of Fitts numerical experiments are untrue.

The proof suggested in Barabanov (1988) of existence of system (1.1) for $n=4$, for which Kalman's conjecture is not satisfied, is an "existence theorem" and requires a careful check. For example, in Bernat \& Llibre (1996); Meisters (1996); Glutsyuk (1998) it is noticed that in the work Barabanov (1988) there are some "gaps" and then in Bernat \& Llibre (1996) an effort was made for their overcoming by using analyticalnumerical methods.

In the present work a constructive algorithm for the construction of classes of systems (1.1), for which Kalman's conjecture is untrue, is suggested.

\section{Harmonic linearization and absolute stability: justification}

After harmonic linearization of system (1.1)

$$
\begin{gathered}
W(p)=r^{*}(P-p I)^{-1} q \\
\operatorname{Im} W\left(i \omega_{0}\right)=0 \\
k=-\left(\operatorname{Re} W\left(i \omega_{0}\right)\right)^{-1}
\end{gathered}
$$

we obtain

$$
\begin{gathered}
\frac{d x}{d t}=P_{0} x+q \psi(\sigma) \\
P_{0}=P+k q r^{*} \\
\varphi(\sigma)=\psi(\sigma)-k \sigma \\
P_{0}: \lambda_{1,2}= \pm i \omega_{0}, \operatorname{Re} \lambda_{j>2}<0
\end{gathered}
$$

If (2.5) is stable for any $\psi(\sigma)=\mu \sigma, \mu \in\left(\mu_{1}, \mu_{2}\right)$, then according tc Aizerman conjecture (2.5) is stable for any $\psi(\sigma)$ such that

$$
\mu_{1} \sigma<\psi(\sigma)<\mu_{2} \sigma, \forall \sigma \neq 0
$$

Applying in this case the method of harmonic balance and describing function method we obtain the existence of periodic solutions

$$
\sigma(t)=r^{*} x(t) \approx a \cos \omega_{0} t
$$

for system (2.5), where $a$ such that

$$
\int_{0}^{2 \pi / \omega_{0}} \psi\left(a \cos \omega_{0} t\right) \cos \omega_{0} t \mathrm{~d} t=k a \int_{0}^{2 \pi / \omega_{0}}\left(\cos \omega_{0} t\right)^{2} \mathrm{~d} t .
$$

However, from here and from condition (2.6) it is follows that $a=0$, since by virtue of stability $k<\mu_{1}, \mu_{2}<k$ is valid. Hence

$$
\begin{array}{r}
k \sigma^{2}<\psi(\sigma) \sigma, \psi(\sigma) \sigma<k \sigma^{2} \\
\forall a \neq 0: \int_{0}^{2 \pi / \omega_{0}}\left(\psi\left(a \cos \omega_{0} t\right) a \cos \omega_{0} t-k\left(a \cos \omega_{0} t\right)^{2}\right) \mathrm{d} t \neq 0
\end{array}
$$

Thus the method of harmonic balance and describing function method confirm the Aizerman's conjecture, but counterexamples are well-known. 


\section{Analytical-numerical method for localization of hidden peri- odic solutions}

We assume first that $\mu_{1}=0, \mu_{2}>0$, the matrix $P$ has two purely imaginary eigenvalues $\pm i \omega_{0}\left(\omega_{0}>0\right)$, and the rest of its eigenvalues have negative real parts. In this case system (1.1) can be represented as

$$
\begin{aligned}
& \dot{x}_{1}=-\omega_{0} x_{2}+b_{1} \varphi\left(x_{1}+c^{*} x_{3}\right) \\
& \dot{x}_{2}=\omega_{0} x_{1}+b_{2} \varphi\left(x_{1}+c^{*} x_{3}\right) \\
& \dot{x}_{3}=A x_{3}+b \varphi\left(x_{1}+c^{*} x_{3}\right) .
\end{aligned}
$$

Here $x_{1}, x_{2} \in \mathbb{R}^{1}, x_{3} \in \mathbb{R}^{n-2}, A$ is a constant $(n-2) \times(n-2)$-matrix, all eigenvalues of which have negative real parts, $b$ and $c$ are constant $(n-2)$-dimensional vectors, $b_{1}$ and $b_{2}$ are certain numbers.

We write now a transfer function of system (3.7) from "input $\varphi$ " to "output $r^{*} x$ ":

$$
W(p)=r^{*}(P-p I)^{-1} q=\frac{-b_{1} p+b_{2} \omega_{0}}{p^{2}+\omega_{0}^{2}}+c^{*}(A-p I)^{-1} b .
$$

Consider the function $\varphi^{0}(\sigma)$ of special form ("saturation"):

$$
\varphi^{0}(\sigma)= \begin{cases}\mu \sigma, & \forall|\sigma| \leq \varepsilon \\ \operatorname{sign}(\sigma) M \varepsilon^{3}, & \forall|\sigma|>\varepsilon\end{cases}
$$

Here $\mu<\mu_{2}, M$ are certain positive numbers, $\varepsilon$ is a small positive parameter.

For system (3.7) with $\varphi=\varphi^{0}$ the following result occurs.

Theorem 1 (Leonov (2010)) If the inequalities

$$
\begin{aligned}
& b_{1}<0, \\
& 0<\mu b_{2} \omega_{0}\left(c^{*} b+b_{1}\right)+b_{1} \omega_{0}^{2}
\end{aligned}
$$

are satisfied, then for small enough $\varepsilon$ system (3.7) with nonlinearity (3.8) has orbitally stable periodic solution, satisfying the following relations

$$
\begin{aligned}
& x_{1}(t)=-\sin \left(\omega_{0} t\right) x_{2}(0)+O(\varepsilon), \\
& x_{2}(t)=\cos \left(\omega_{0} t\right) x_{2}(0)+O(\varepsilon), \\
& x_{3}(t)=O(\varepsilon), \\
& x_{1}(0)=O\left(\varepsilon^{2}\right), \\
& x_{2}(0)=-\sqrt{\frac{\mu\left(\mu b_{2} \omega_{0}\left(c^{*} b+b_{1}\right)+b_{1} \omega_{0}^{2}\right)}{-3 \omega_{0}^{2} M b_{1}}}+O(\varepsilon), \\
& x_{3}(0)=O\left(\varepsilon^{2}\right) .
\end{aligned}
$$

The methods for the proof of this theorem are developed in Leonov et al. (1996); Leonov (2009, 2010).

Consider for $j=1, \ldots, m$ a finite sequence of functions

$$
\varphi^{j}(\sigma)=\left\{\begin{array}{ll}
\mu \sigma, & \forall|\sigma| \leq \varepsilon_{j} ; \\
\operatorname{sign}(\sigma) M \varepsilon_{j}^{3}, & \forall|\sigma|>\varepsilon_{j} .
\end{array} \quad \varepsilon_{j}=\frac{j}{m} \sqrt{\frac{\mu}{M}}\right.
$$

We choose $m$ in such a way that the graphs of functions $\varphi^{j}$ and $\varphi^{j+1}$ are slightly distinct from each other outside small neighborhoods of points of discontinuity. 
Theorem 1 permits one to find the close to harmonic one stable periodic solution $x(t)=x^{0}(t)$ of system

$$
\frac{d x}{d t}=P x+q \varphi^{j}\left(r^{*} x\right)
$$

with $j=0$. All the points of this periodic solution may be placed in domain of attraction of stable solution $x^{1}(t)$ of system (3.11) with $j=1$ or in change from system (3.11) with $j=0$ to system (3.11) with $j=1$ we observe the bifurcation of instability and vanishing periodic solution. In the first case it is possible to find $x^{1}(t)$ numerically, starting a trajectory of system (3.11) with $j=1$ from the initial point $x^{0}(0)$.

Starting from the point $x^{0}(0)$, after transient process the computational procedure reaches the periodic solution and computes it. In this case the interval $[0, T]$ on which the computation is carried out, must be sufficiently large.

After the computation of $x^{1}(t)$ it is possible to take system (3.11) with $j=2$ and to organize a similar procedure of computing the periodic solution $x^{2}(t)$ by starting from the initial point $x(0)=x^{1}(T)$ a trajectory, which with increasing $t$ may reach the periodic trajectory $x^{2}(t)$ or we observe the instability bifurcation destroying periodic solution.

Proceeding this procedure and computing sequentially the periodic solutions $x^{j}(t)$, using trajectories of system (3.11) with the initial data $x^{j}(0)=x^{j-1}(T)$, we either obtain a periodic solution of system (3.11) with $j=m$, either observe, at a certain step, the instability bifurcation destroying periodic solution and stop algorithm.

Suppose that the periodic solution $x^{m}(t)$ of system (3.11) with monotone and continuous function $\varphi^{m}(\sigma)$ is computed. In this case we organize a similar computational procedure for the sequence of systems

$$
\frac{d x}{d t}=P x+q \psi^{i}\left(r^{*} x\right)
$$

Here $i=0, \ldots, h, \quad \psi^{0}(\sigma)=\varphi^{m}(\sigma)$ and

$$
\psi^{i}(\sigma)= \begin{cases}\mu \sigma, & \forall|\sigma| \leq \varepsilon_{m} \\ i\left(\sigma-\operatorname{sign}(\sigma) \varepsilon_{m}\right) N+\operatorname{sign}(\sigma) \mu \varepsilon_{m}, & \forall|\sigma|>\varepsilon_{m}\end{cases}
$$

were $N$ is a certain positive parameter such that $h N<\mu_{2}$.

The finding of periodic solutions $x^{i}(t)$ of system (3.12) gives a certain counterexample to Kalman's conjecture for each $i=1, \ldots, h$.

We now give the corresponding examples.

\section{Counterexamples to Kalman problem}

Example 1. Consider a system

$$
\begin{aligned}
& \dot{x}_{1}=-x_{2}-10 \varphi\left(x_{1}-10.1 x_{3}-0.1 x_{4}\right) \\
& \dot{x}_{2}=x_{1}-10.1 \varphi\left(x_{1}-10.1 x_{3}-0.1 x_{4}\right) \\
& \dot{x}_{3}=x_{4} \\
& \dot{x}_{4}=-x_{3}-x_{4}+\varphi\left(x_{1}-10.1 x_{3}-0.1 x_{4}\right)
\end{aligned}
$$

Here for $\varphi(\sigma)=k \sigma$, linear system (4.13) is stable for $k \in(0,9.9)$ and by the above-mentioned theorem for piecewise-continuous nonlinearity $\varphi(\sigma)=\varphi^{0}(\sigma)$ with sufficiently small $\varepsilon$ there exists periodic solution.

Now we make use the algorithm of constructing periodic solutions. Suppose $\mu=M=1, \varepsilon_{1}=0.1, \varepsilon_{2}=$ $0.2, \ldots, \varepsilon_{10}=1$. For $j=1, \ldots, 10$, we construct sequentially solutions of system (4.13), assuming that by (3.10) the nonlinearity $\varphi(\sigma)$ is equal to $\varphi^{j}(\sigma)$. Here for all $\varepsilon_{j}, j=1, \ldots, 10$ there are periodic solutions. 
At the first step for $j=0$ by the theorem the initial data of stable periodic oscillation take the form:

$$
\begin{aligned}
& x_{1}(0)=O(\varepsilon), x_{3}(0)=O(\varepsilon), x_{4}(0)=O(\varepsilon), \\
& x_{2}(0)=-1.7513+O(\varepsilon) .
\end{aligned}
$$

Therefore for $j=1$ a trajectory starts from the point $x_{1}(0)=x_{3}(0)=x_{4}(0)=0, x_{2}(0)=-1.7513$. The projection of this trajectory on the plane $\left(x_{1}, x_{2}\right)$ and the output of system $r^{*} x(t)=x_{1}(t)-10.1 x_{3}(t)-0.1 x_{4}(t)$ are shown in Fig. 1.
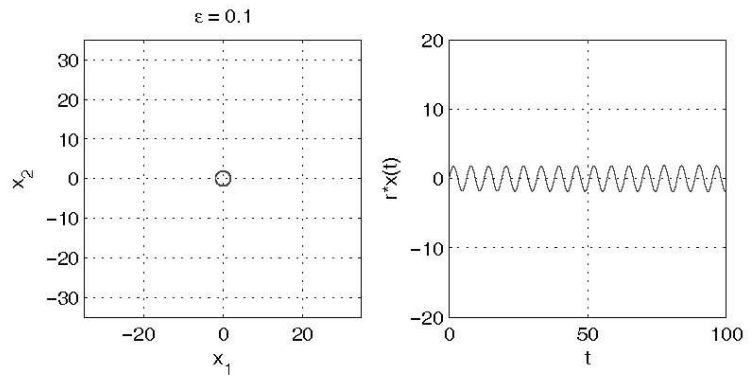

Figure 1: $\quad x_{1}(0)=x_{3}(0)=x_{4}(0)=0, x_{2}(0)=-1.7513$

From the figure it follows that after transient process stable periodic solution is reached. At the first step the computational procedure is ended at the point $x_{1}(T)=0.7945, x_{2}(T)=1.7846, x_{3}(T)=0.0018, x_{4}(T)=$ -0.0002 , where $T=1000 \pi$.

Further, for $j=2$ we take the following initial data: $x_{1}(0)=0.7945, x_{2}(0)=1.7846, x_{3}(0)=0.0018, x_{4}(0)=$ -0.0002 , and obtain periodic solutions (Fig. 2).

Figure 2: $\quad$ step 2: $x_{1}(0)=0.7945, x_{2}(0)=1.7846, x_{3}(0)=0.0018, x_{4}(0)=-0.0002$

Proceeding this procedure for $j=3, \ldots 10$, we sequentially approximate (Fig. 3.9) a periodic solution of system (4.13) (Fig. 10).

Figure 3: $\quad$ step 3: $x_{1}(0)=-2.4889, x_{2}(0)=-0.9193, x_{3}(0)=-0.0133, x_{4}(0)=-0.0033$

Figure 4: $\quad$ step 4: $x_{1}(0)=3.8129, x_{2}(0)=0.5056, x_{3}(0)=0.0416, x_{4}(0)=0.013$

Figure 5: $\quad$ step 5: $x_{1}(0)=3.8854, x_{2}(0)=-4.3572, x_{3}(0)=0.0342, x_{4}(0)=0.0938$

Note that for $\varepsilon_{j}=1$ the nonlinearity $\varphi_{j}(\sigma)$ is monotone. The computational process is ended at the point $x_{1}(T)=1.6193, x_{2}(T)=-29.7162, x_{3}(T)=-0.2529, x_{4}(T)=1.2179$, where $T=1000 \pi$. 
Figure 6: $\quad$ step 6: $x_{1}(0)=-4.6043, x_{2}(0)=6.802, x_{3}(0)=-0.0536, x_{4}(0)=-0.1753$
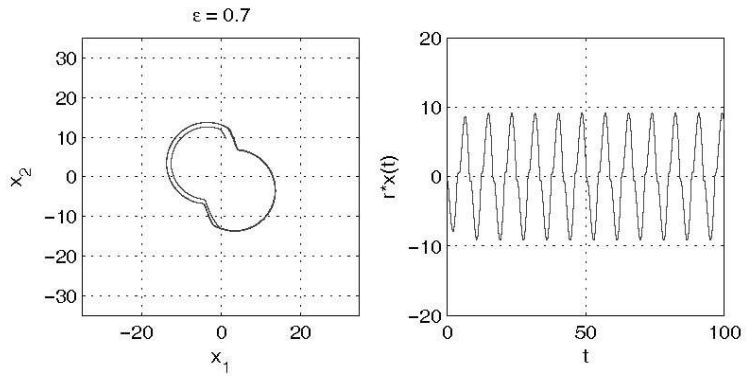

Figure 7: $\quad$ step 7: $x_{1}(0)=1.1209, x_{2}(0)=9.7293, x_{3}(0)=0.1817, x_{4}(0)=-0.3342$

Figure 8: $\quad$ step 8: $x_{1}(0)=-4.0482, x_{2}(0)=-7.4042, x_{3}(0)=-0.4370, x_{4}(0)=0.1263$

Figure 9: $\quad$ step 9: $x_{1}(0)=-10.9852, x_{2}(0)=-6.2842, x_{3}(0)=-0.6842, x_{4}(0)=0.0833$
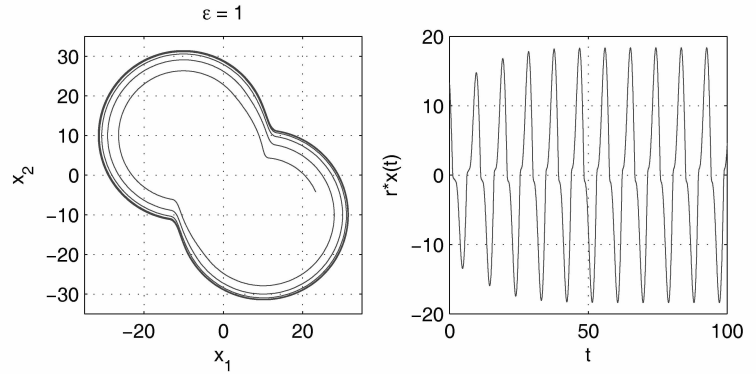

Figure 10: $\quad$ step 10: $x_{1}(0)=23.3012, x_{2}(0)=-4.2240, x_{3}(0)=0.9856, x_{4}(0)=0.0905$ 
We also remark that if in place of sequential increasing $\varepsilon^{j}$ it is computed a solution with initial data according to (3.9) for $\varepsilon=1$, then the solution "fall down" to zero.

Change the nonlinearity $\varphi(\sigma)$ to the strictly increasing function $\psi^{i}(\sigma)(3.12)$, where $\mu=1, \varepsilon_{m}=1, N=$ 0.01 , for $\mathrm{i}=1, \ldots, 5$, and, continue the sequential construction of periodic solutions for system (4.13). The graph of such nonlinearity is shown in Fig. 11.

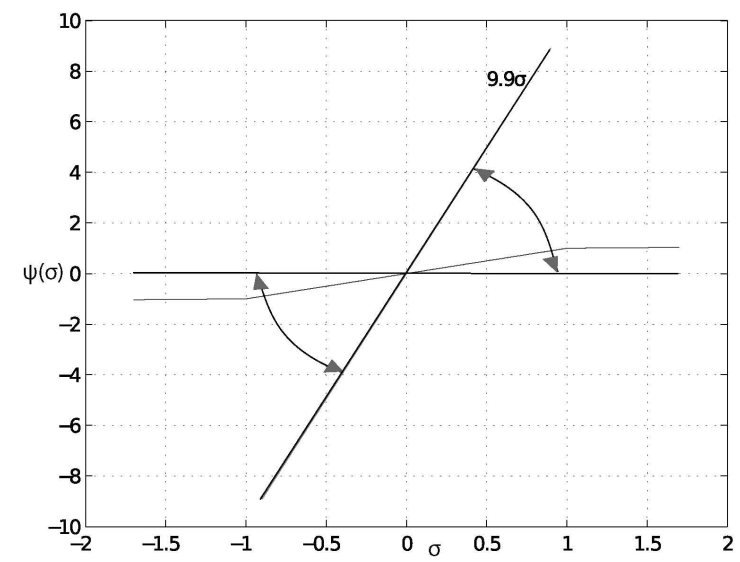

Figure 11: The graph of $\psi^{i}(\sigma)$ for $\mathrm{i}=5$ and stability sector

The periodic solutions obtained are shown in Fig. 12-16.
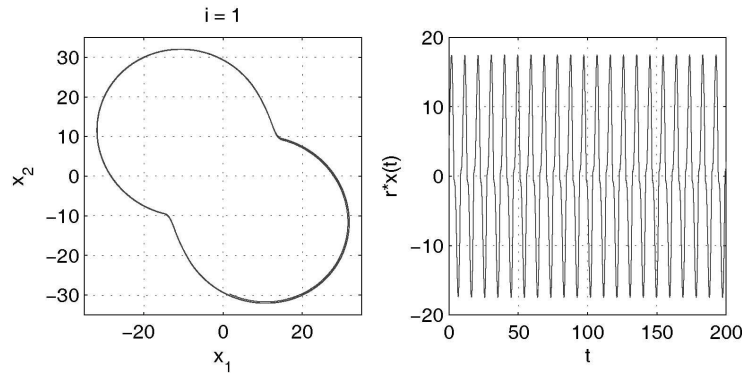

Figure 12: $\quad x_{1}(0)=1.6193, x_{2}(0)=-29.7163, x_{3}(0)=-0.2529, x_{4}(0)=1.2179$
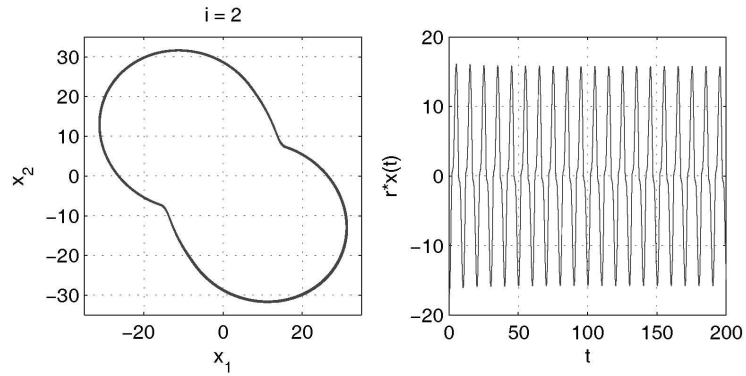

Figure 13: $x_{1}(0)=-29.6754, x_{2}(0)=20.8277, x_{3}(0)=-1.2737, x_{4}(0)=-0.4976$ 

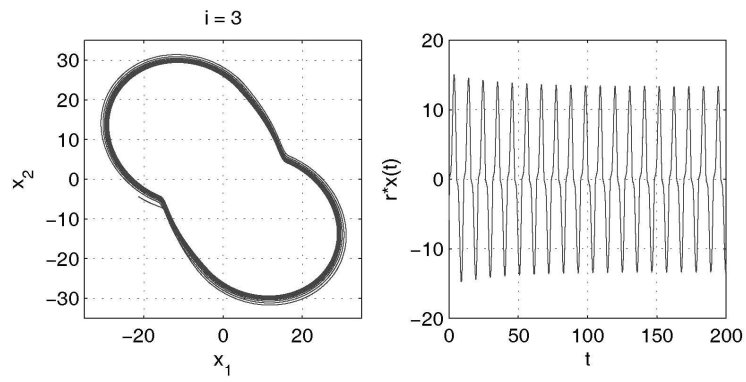

Figure 14: $x_{1}(0)=-21.3740, x_{2}(0)=-4.3702, x_{3}(0)=-1.5408, x_{4}(0)=0.1792$
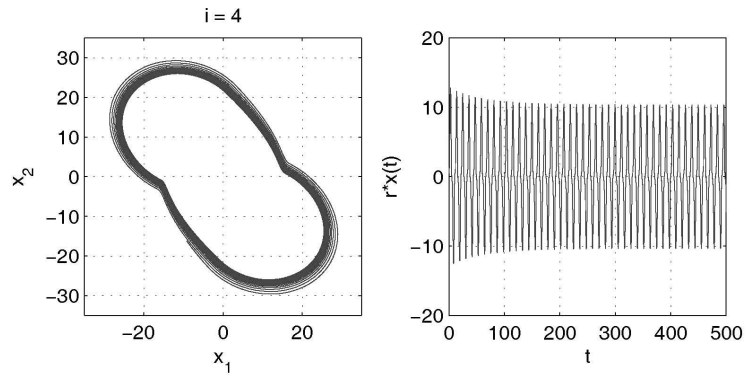

Figure 15: $x_{1}(0)=-9.1968, x_{2}(0)=-16.1835, x_{3}(0)=-0.9760, x_{4}(0)=0.9528$
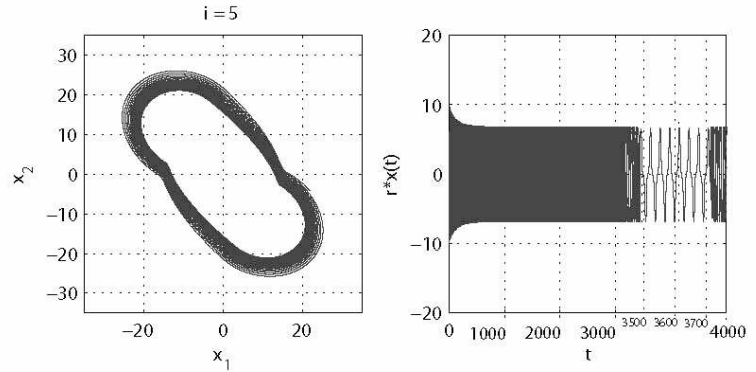

Figure 16: $x_{1}(0)=21.9632, x_{2}(0)=-4.2469, x_{3}(0)=1.5638, x_{4}(0)=-0.0774$
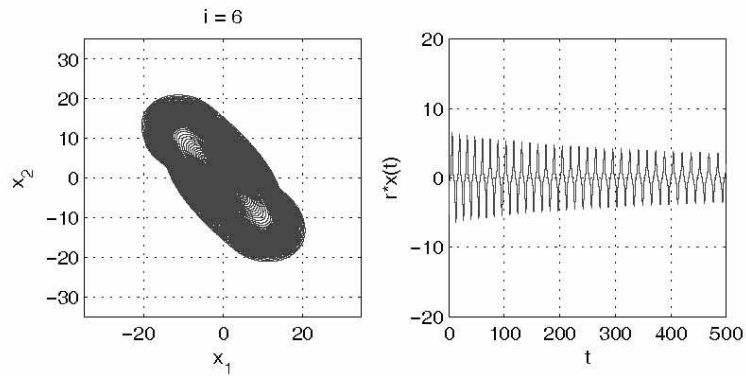

Figure 17: $x_{1}(0)=-19.6256, x_{2}(0)=8.3259, x_{3}(0)=-1.4322, x_{4}(0)=0.0047$ 
In the case of the computation of solution for $i=6$ there occurs the vanishing of periodic solution (Fig. 17).

Example 2. For system (4.13) with smooth strictly increasing nonlinearity

$$
\phi(\sigma)=\tanh (\sigma)=\frac{e^{\sigma}-e^{-\sigma}}{e^{\sigma}+e^{-\sigma}}
$$

there exists a periodic solution (Fig. 18). Here

$$
0<\frac{\mathrm{d}}{\mathrm{d} \sigma} \tanh (\sigma) \leq 1, \forall \sigma
$$
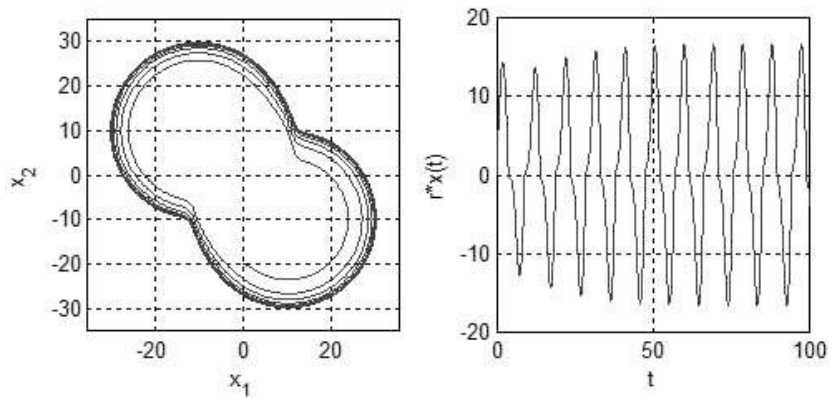

Figure 18: The projection of trajectory with the initial data $x_{1}(0)=x_{3}(0)=x_{4}(0)=0, x_{2}(0)=-20$ of system (4.14) on the plane $\left(x_{1}, x_{2}\right)$

\section{References}

M.A. Aizerman. On a problem concerning the stability "in the large" of dynamical systems. Russian mathematical surveys, Vol. 4, Iss. 4, pp. 186-188, 1949.

N.E. Barabanov. On the Kalman problem Sib. Math. J., 29(3), pp. 333-341, 1988.

J. Bernat, J. Llibre. Counterexample to Kalman and Markus-Yamabe conjectures in dimension larger than 3. Dynamics of Continuous, Discrete and Impulsive Systems, Vol. 2, N3. pp. 337-379, 1996.

R.E. Fitts. Two counterexamples to Aizerman's conjecture Trans. IEEE., V. AC-11, N3, pp. 553-556, 1966.

Kalman R.E. Physical and Mathematical mechanisms of instability in nonlinear automatic control systems Transactions of ASME 1957. v.79 N 3 P. 553-566.

G.A. Leonov, I.M. Burkin, A.I. Shepeljavyi. Frequency methods in oscillation theory. Kluwer Academic Publishers, 1996.

G.A. Leonov. On Aizerman problem. Automation and remote control, N7, pp. 37-49, 2009.

G.A. Leonov. Effective methods for periodic oscillations search in dynamical systems. App. math. $\&$ mech., Vol. 74, Iss. 1, pp. 37-73, 2010.

G. Meisters. A Biography of the Markus-Yamabe Conjecture, http://www.math.unl.edu/ gmeisters1/papers/HK1996.pdf, 1996.

Meetings of the Moscow Mathematical Society. Russian mathematical surveys, 53:2(320), pp. 169-170, 1998.

Meetings of the Leningrad Mathematical Society. Russian mathematical surveys, 37:1(223), pp. $164,1982$. 